\title{
Elevated levels of matrix metalloproteinases in bronchoalveolar lavage fluid of emphysematous patients
}

Geraldine A Finlay, Kenneth J Russell, Kevin J McMahon, Elizabeth M D'arcy, James B Masterson, Muiris X FitzGerald, Clare M O'Connor

\begin{abstract}
Background - Matrix degradation in emphysema has long been attributed to the action of neutrophil elastase (NE). More recently a role for other proteases, particularly the matrix metalloproteinases (MMPs), in the pathogenesis of this disease has been proposed. To date, however, the presence of MMPs in the lungs of patients with emphysema has not been demonstrated.
\end{abstract}

Methods - Samples of bronchoalveolar lavage (BAL) fluid from 10 patients with emphysema and from control subjects matched for sex and current smoking status were assessed for collagenase, gelatinase, and NE activity. Pulmonary function tests and computed tomographic (CT) scans were carried out on all study subjects.

Results - Collagenase activity was detected in BAL fluid samples from all emphysematous patients but in only one smoking control $(p<0.001)$. Gelatinase B was present in six patients and in two smoking controls $(p<0.03)$. The concomitant presence of gelatinase $B$ in complex with lipocalin (NGAL) in the gelatinase positive samples suggests that the neutrophil is a significant source of the gelatinase $B$ observed. NE was detected in six of the 10 patients with emphysema and in two smoking controls $(p<0.01)$, indicating that collagenase was more useful in discriminating between disease and control groups than either $\mathrm{NE}$ or gelatinase B. No relationship was observed between any of the enzymes measured and pulmonary function or CT density score. Conclusions - This study demonstrates, for the first time, the presence of increased levels of matrix metalloproteinases in the lungs of patients with emphysema and suggests that, in BAL fluid, collagenase activity may be a better indicator of the presence of emphysema than elastase.

(Thorax 1997;52:502-506)

Keywords: emphysema, matrix metalloproteinases, neutrophil elastase.
Pulmonary emphysema is a condition of the lung characterised by progressive destruction and loss of functioning alveoli. The mechanisms involved in extracellular matrix (ECM) damage are not precisely known but a significant body of evidence supports the proteaseantiprotease theory which postulates that an excess of proteolytic activity over the inhibitory capacity of the lung leads to parenchymal destruction. ${ }^{1}$ Most studies to date have focused on the role of neutrophil elastase (NE) as the major effector protease involved. ${ }^{2}$ More recently, however, there has been considerable speculation on the potential involvement of other proteases, particularly the matrix metalloproteinases (MMPs), in matrix degradation in emphysema. ${ }^{3}$

The MMPs are a highly homologous family of endopeptidases which are collectively capable of breaking down all the constituents of the alveolar ECM, including collagen, elastin, proteoglycans, laminin, and fibronectin. ${ }^{4}$ They are produced by a range of stromal cells and by two of the major inflammatory cells implicated in emphysema - the alveolar macrophage and the neutrophil. Indirect evidence that MMPs may play a part in tissue destruction in emphysema comes from a number of studies. Janoff et al demonstrated that the elastolytic activity of bronchoalveolar lavage (BAL) fluid from smokers was significantly inhibited by metal chelating agents and concluded that MMPs were responsible for almost $50 \%$ of the activity measured. ${ }^{5}$ More recently, D'Armiento et al have described the development of emphysema in transgenic mice expressing human interstitial collagenase (MMP-1) in their lungs, lending support to the theory that MMPs contribute to the pathogenesis of emphysema. ${ }^{6}$ To date, however, the presence of MMPs in the lungs of patients with emphysema has not been demonstrated.

The aim of this study was to determine whether increased quantities of MMPs are present in the lungs of patients with emphysema. Levels of two of the major MMPs, collagenase and gelatinase, were assessed in epithelial lining fluid (ELF) from patients with emphysema and compared with levels in a control group. The level of NE in the ELF was also assessed in both groups. 


\section{Methods}

STUDY POPULATION

Ten patients with emphysema (all men, four smokers and six ex-smokers) aged 43-75 years were entered into the study. Criteria for selection of emphysema patients were (1) impaired pulmonary function as indicated by a forced expiratory volume in one second $\left(\mathrm{FEV}_{1}\right)$ $<75 \%$ of predicted normal and/or a ratio of $\mathrm{FEV}_{1}$ to forced vital capacity (FVC) of $<75 \%$, (2) high resolution computed tomographic (CT) evidence of emphysema, (3) clinical history, medical symptoms, and chest radiograph consistent with the diagnosis of emphysema, and (4) no reversibility of airflow obstruction after inhalation of $\beta_{2}$ agonists. None of the patients had received inhaled or oral steroids at the time of lavage or in the previous six weeks.

Eleven control subjects (all men, five smokers and six non-smokers) aged $43-58$ years undergoing a brief general anaesthetic for minor surgery were also recruited to the study. To exclude the presence of emphysema clinical history, medical examination, chest radiography, pulmonary function tests and CT scans were carried out on all control subjects.

No subject in either the patient or control group had a history of atopy or episodic wheezing, nocturnal dyspnoea or ankle oedema. All subjects denied having significant symptoms of a respiratory tract infection in the preceding six weeks. Arterial blood gas tensions measured in all subjects whilst breathing room air showed no evidence of clinically significant hypoxaemia or hypercapnia and the serum levels of $\alpha_{1}$ proteinase inhibitor were normal in all subjects. All current smokers were lifelong smokers of at least 10 cigarettes per day and ex-smokers had ceased their habit for at least 10 years. Smokers were asked to refrain from smoking for at least 12 hours prior to lavage. Thus, in each case the diagnosis of emphysema was established or excluded and the absence of coexistent disease confirmed. All subjects gave their informed consent and the project was approved by St Vincent's Hospital ethics committee.

\section{BRONCHOSCOPIC EXAMINATION AND}

BRONCHOALVEOLAR LAVAGE

Before bronchoscopic examination patients were premedicated with intramuscular atropine $(0.6 \mathrm{mg})$. The upper respiratory tract was then anaesthetised with nebulised lignocaine, patients sedated with intravenous midazolam $(10 \mathrm{mg})$, and bronchoalveolar lavage was performed as previously described. ${ }^{7}$ Oxygen was provided for emphysematous patients by nasal catheter at $21 / \mathrm{min}$ during bronchoscopy and electrocardiographic rhythm was continuously monitored. Control subjects received intravenous suxamethonium and propafol and were rapidly intubated and anaesthetised with isofluorane, immediately following which bronchoscopy was performed prior to any surgical procedure.

Samples of BAL fluid were processed under sterile conditions within one hour of collection.
Viability of cells recovered by centrifugation was over $90 \%$ in all cases. Differential counts were determined on Diff-Quick stained preparations. Cell-free BAL fluid was divided into aliquots for measurement of collagenase, gelatinase, and NE. To avoid proteolysis following sampling the serine proteinase inhibitors diisopropylfluorophosphate (DIPF, $1 \mathrm{mM}$ ) and phenyl-methyl-sulphonylfluoride (PMSF, $1 \mathrm{mM}$ ) were added to the aliquots to be analysed for MMP activity. No inhibitors were added to the samples to be assessed for the presence of NE. All samples were stored at $-70^{\circ} \mathrm{C}$ until analysis which was performed within six months of collection. For standardisation purposes the urea concentration in the serum and BAL fluid was measured in all subjects, the volume of dilution was determined, and the volume of epithelial lining fluid (ELF) was calculated. ${ }^{8}$ Enzyme results were expressed per $\mathrm{ml}$ ELF.

\section{ENZYMATIC ANALYSIS}

For measurement of collagenase activity samples of BAL fluid were concentrated $\times 20$ by ultrafiltration on CF25 Centriflo membrane cones (Amicon Corporation, Danvers, Massachusetts, USA). Latent collagenase was activated by incubation with $1 \mathrm{mM} p$-aminophenylmercuric acetate (APMA) at $35^{\circ} \mathrm{C}$ for 10 minutes and collagenase activity was then determined by measuring the release of radiolabelled fragments from ${ }^{14} \mathrm{C}$-labelled type I collagen as previously described. ${ }^{7}$ Gelatin zymography was carried out on unconcentrated samples of BAL fluid as described by Overall et al. ${ }^{9}$ Positive standards included a neutrophil lysate and a preparation containing gelatinase $A$ and $B$ from gelatinase B transfected $\mathrm{HK}$ cells (obtained from Dr S McDonnell, Dublin City University, Dublin, Ireland). Densitometry was carried out on negative images of the zymograms using the GDS- 8000 Complete Gel Imaging System (Phoretix International, Newcastle upon Tyne, UK). The density of bands in individual samples was standardised to the gelatinase $\mathrm{A} /$ gelatinase $\mathrm{B}$ positive standard on the corresponding zymogram. Units of gelatinase activity were expressed as arbitrary densitometric units per ml ELF. To assess NE activity, samples of lavage fluid were concentrated $\times 20$ by centrifugal evaporation (Gyrovap, Howe and Co, UK) and NE activity was measured using the specific chromogenic peptide substrate, N-methoxy-succinyl-AlaAla-Pro-val $p$-nitroanilide (MEOSAAPVPNA, Sigma, Poole, UK) at a concentration of $4.2 \mathrm{mM}$. Using an extinction coefficient of $8800 / 1 / \mathrm{mol} / \mathrm{cm}$ molar concentrations of peptide hydrolysed were determined and enzyme units, defined as the release of $1 \mathrm{M} p$-nitroanilide $/ \mathrm{ml} /$ min of sample, were calculated.

\section{STATISTICAL ANALYSIS}

The non-parametric Wilcoxon's rank sum test was used for statistical analysis of cellular and enzyme data. Regression analysis was used to correlate clinical, radiological and enzyme data. 

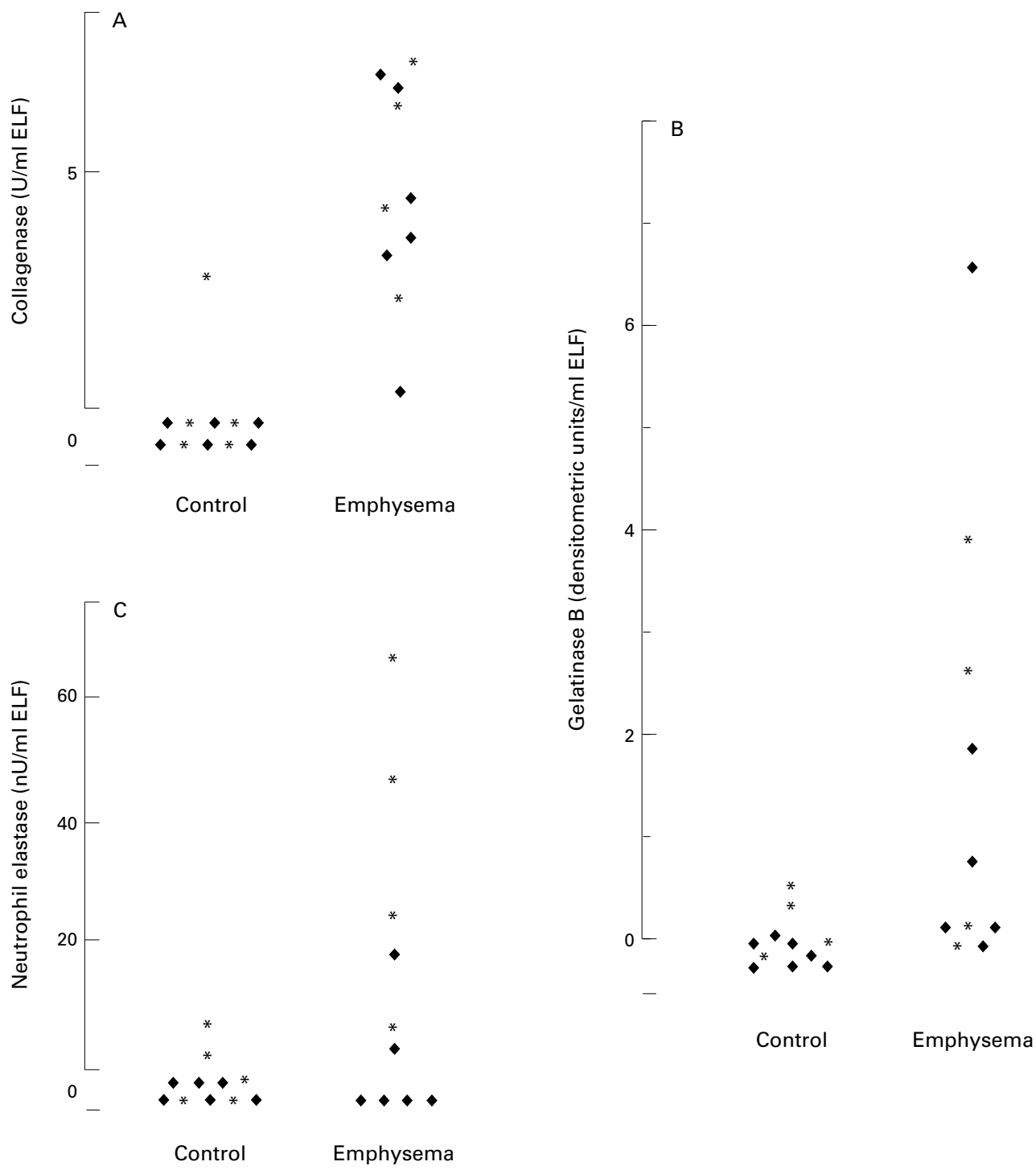

Figure 1 Levels of $(A)$ collagenase, $(B)$ gelatinase B, and $(C)$ neutrophil elastase in the epithelial lining fluid (ELF) of patients with emphysema and a control population. ${ }^{*}=$ current smoker; $\bullet=n o n-s m o k e r$ or ex-smoker. Levels are expressed as units per $m l$ ELF.

Values were expressed as medians with absolute ranges unless otherwise stated. In all tests $p$ values of $<0.05$ were taken as indicating a significant difference between the groups.

\section{Results}

BAL FLUID CELL PROFILE

The total number of cells recovered in the BAL fluid did not differ between patients with emphysema (median $12.9 \times 10^{6}$; range $1.5-36.4 \times$ $10^{6}$ cells) and controls (median $10.0 \times 10^{6}$; range $1.0-37.4 \times 10^{6}$ cells). In both groups over $90 \%$ of cells recovered were alveolar macrophages (91.8\% and $91.4 \%$ in emphysematous and control groups, respectively) and less than $2 \%$ were neutrophils $(1.35 \%$ and $1.2 \%$ for emphysematous and control groups, respectively).

COLLAGENASE ACTIVITY

Collagenase was detected in all 10 patients with emphysema (median 4.41, range 1.04-6.86 U/ $\mathrm{ml} \mathrm{ELF}$ ) but in only one control subject (3.1 U/ $\mathrm{ml} \mathrm{ELF}$; $<<0.001$; fig $1 \mathrm{~A})$. There was no statistical difference in collagenase levels between smokers and non-smokers in the patient group, nor was the presence of collagenase in ELF related to age or the number of neutrophils or macrophages in the BAL fluid. No correlation was seen between the collagenase level and CT density score or pulmonary function.

GELATINASE ZYMOGRAPHY

Gelatinase was present in ELF from five patients with emphysema and two control subjects, both of whom were smokers (fig 1B). In both control and patient groups gelatin zymography showed the presence of four distinct gelatinase bands of molecular weights $92 \mathrm{kDa}$ (corresponding to latent gelatinase $\mathrm{B}$ ), $130 \mathrm{kDa}, 220 \mathrm{kDa}$, and $270 \mathrm{kDa}$ (fig 2). This banding pattern corresponded to that observed for the standard neutrophil lysate and is highly 


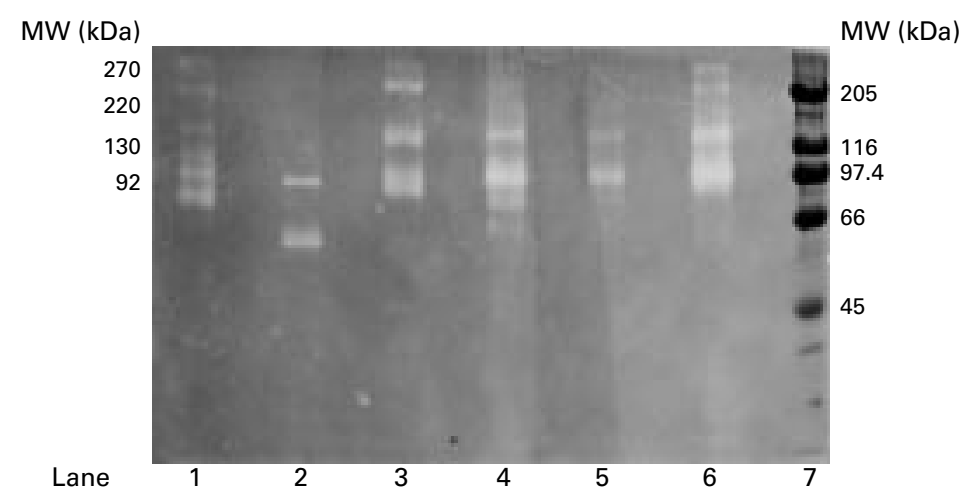

Figure 2 A typical gelatinase zymogram of samples of bronchoalveolar lavage (BAL) fluid from patients with emphysema. Lane 1: neutrophil lysate (positive standard) demonstrating four distinct bands of gelatinase present at $270 \mathrm{kDa}, 220 \mathrm{kDa}$ (multiple forms of $92 \mathrm{kDa}$ gelatinase B), $130 \mathrm{kDa}$ (neutrophil gelatinase associated lipocalin complex; NGAL), and $92 \mathrm{kDa}$ (latent form of gelatinase B); lane 2: positive standard of latent gelatinase $B$ at $92 \mathrm{kDa}$ and gelatinase $A$ at $72 \mathrm{kDa}$; lanes 3-6: samples of $B A L$ fluid from patients with emphysema with bands of gelatinase present at $270 \mathrm{kDa}$, $220 \mathrm{kDa}, 130 \mathrm{kDa}$, and $92 \mathrm{kDa}$; lane 7: molecular weight (MW) markers.

characteristic of gelatinase released from neutrophils. ${ }^{10}$ The band of molecular weight $130 \mathrm{kDa}$ has been characterised as gelatinase B in complex with another neutrophil secondary granule protein, lipocalin (neutrophil gelatinase associated lipocalin; NGAL), ${ }^{11}$ and the higher molecular weight bands as multiple forms of gelatinase $\mathrm{B} .{ }^{10}$ In four patient samples additional bands (not shown) were observed at $72 \mathrm{kDa}$, which corresponds to the molecular weight of latent gelatinase $\mathrm{A}$, and $63 \mathrm{kDa}$ which may represent the active form of gelatinase A. Densitometric quantitation showed significantly higher levels of gelatinase $\mathrm{B}(92 \mathrm{kDa})$ in patients (median 0.32; range 0-6.51 densitometric units/ml ELF) compared with controls (median 0; range 0-0.44 densitometric units/ml ELF; $\mathrm{p}<0.03$ ). Within the patient group there was no difference in gelatinase $B$ levels between smokers and ex-smokers.

\section{NEUTROPHIL ELASTASE ACTIVITY}

Six patients with emphysema had demonstrable levels of free NE activity in their ELF (median 6.05 , range $0-68.56 \mathrm{nU} / \mathrm{ml}$; fig $1 \mathrm{C}$ ). Within the patient group the level of NE was significantly higher in smokers (median 38.3, range $6.9-68.56 \mathrm{nU} / \mathrm{ml} \mathrm{ELF}$ ) than in non-smokers (median 0.00, range $0.0-22.4 \mathrm{nU} / \mathrm{ml} \mathrm{ELF}$; $\mathrm{p}<0.02)$. NE activity was detected in only two control subjects (2.84 and $8.8 \mathrm{nU} / \mathrm{ml} \mathrm{ELF})$, both of whom were smokers. No correlation was observed between NE activity and pulmonary function or CT lung density, nor was a relationship noted with neutrophil or macrophage numbers in the BAL fluid.

\section{Discussion}

This study provides, for the first time, direct evidence of the presence of increased quantities of the MMPs collagenase and gelatinase in the epithelial lining fluids of emphysematous patients compared with healthy controls. All of the patients studied had demonstrable ELF collagenase activity, while collagenase was de- tected in the ELF from only one control subject, which suggests that ELF collagenase may be a useful discriminator for the presence of emphysema.

The origin of the collagenase activity detected in the lavage fluids of these patients is not clear. Collagenase activity was determined by degradation of a radiolabelled type I collagen substrate which is preferentially degraded by neutrophil collagenase (MMP-8). ${ }^{12}$ While interstitial collagenase (MMP-1) - which is produced by alveolar macrophages and interstitial cells - can also degrade type I collagen, it does so at a rate which is unlikely to be detected in the 90 minute incubation period of the assay employed. It is therefore likely that the collagenase activity measured was that of neutrophil collagenase.

Of the patients studied, $50 \%$ had detectable gelatinase in their epithelial lining fluids compared with $18 \%$ of controls. Zymographic profiles indicated that the bulk of gelatinase present in ELF is attributable to gelatinase B (MMP-9). Zymography also demonstrated the presence of NGAL and multiple forms of gelatinase $\mathrm{B}$, a banding pattern strongly characteristic of gelatinase $\mathrm{B}$ of neutrophil origin. ${ }^{1011}$ However, gelatinase $\mathrm{B}$ can also be secreted by alveolar macrophages and the possibility that the macrophage is another source of ELF gelatinase B in emphysema cannot be ruled out. Furthermore, gelatinase $\mathrm{A}$, which is produced by interstitial cells and macrophages, was present in the ELF of four patients with emphysema.

Six of the 10 patients and two of the 11 controls had detectable NE activity in the ELF. Thus, the ability of NE to discriminate between an emphysematous patient and a healthy control was not as accurate as that observed for ELF collagenase.

These observations must be taken in the context of synergistic modulation of extracellular matrix proteolysis. In vitro, serine proteases activate latent forms of MMPs. ${ }^{13} \mathrm{NE}$ can also inactivate tissue inhibitor of metalloproteinases and MMPs can inactivate $\alpha_{1}-\mathrm{PI}$, a major inhibitor of $\mathrm{NE},{ }^{14}$ which suggests that interaction between MMPs and the serine proteases may be of importance in the unchecked digestion of extracellular matrix. Given that only $10-15 \%$ of smokers develop emphysema, ${ }^{15}$ it could be speculated that production and release of all three proteases in conjunction with each other may be one of the biological variables which renders an individual vulnerable to the development of progressive lung disease subsequent to cigarette smoking.

In conclusion, this study highlights the potential contribution of the matrix metalloproteinases collagenase and gelatinase $\mathrm{B}$, in addition to neutrophil elastase, in the pathogenesis of human emphysema. It is likely that the interaction of these potent proteases is involved in the irreversible and progressive alveolar destruction which characterises emphysema.

This work was supported by the Health Research Board of Ireland.

1 Snider GL. Emphysema: the first two centuries and beyond Part II. Am Rev Respir Dis 1992;146:1615-22. 
2 Tetley TD. Proteinase imbalance: its role in lung disease. Thorax 1993;48:560-5.

3 Shapiro SD. Elastolytic metalloproteinases produced by human mononuclear phagocytes. Potential roles in destructive lung disease. Am $\mathcal{F}$ Respir Crit Care Med 1994; 150:S160-4

4 O'Connor CM, FitzGerald MX. Matrix metalloproteases and lung disease. Thorax 1994;49:101-8.

5 Janoff A, Raju R, Dearing LR. Levels of elastase activity in bronchoalveolar lavage fluids of healthy smokers and nonsmokers. Am Rev Respir Dis 1983;127:540-4

6 D'Armiento J, Dalal SS, Okada Y, Berg RA, Chada K. Collagenase expression in the lungs of transgenic mice causes pulmonary emphysema. Cell 1992;71:955-61.

7 O'Connor C, Odlum A, Van Breda A, Power C, FitzGerald MX. Collagenase and fibronectin in patients with sarcoidosis. Thorax 1988;43:393-400.

8 Rennard SJ, Basset G, Lecossier D, O'Donnell KM, Pinkston P, Martin PG, et al. Estimation of the volume of epithelial lining fluid recovered by lavage using urea as a marker of dilution. $\mathcal{A}$ Appl Physiol 1986;60:532-8.

9 Overall CM, Wrana JL, Sodek J. Independent regulation of collagenase, $72-\mathrm{kD}$ a progelatinase, and metalloproteinase inhibitor expression in human fibroblasts by transforming growth factor- $\beta$. F Biol Chem 1989;264:1860-9.

10 Hibbs MS, Hasty KA, Seyer JM, Kang AH, Mainardi CL. Biochemical and immunological characterisation of the secreted forms of human neutrophil gelatinase. $7 \mathrm{Bio}$ Chem 1985;260:2493-500.

11 Kjeldsen L, Johnson AH, Sengeløv H, Borregaard N. Isolation and primary structure of NGAL, a novel protein 268: $10425-32$.

12 Horwitz AL, Hance AJ, Crystal RG. Granulocyte collagenase: selective degradation of type I relative to type

13 Hubbard RC, Brantley ML, Crystal RG. Proteases. In: Hubbard RC, Brantley ML, Crystal RG. Proteases. In:
Crystal RG, West JD, eds. The lung. New York: Raven Press, 1991:1763-73.

14 Okada Y, Watanabe S, Nakanishi I, Kishi J, Hayakama T, Watorek W. Inactivation of tissue inhibitor of metalloproteinases by neutrophil elastase and other serine proteases. FEBS Lett 1988;229:157-60.

15 Sherman CB. Health effects of cigarette smoking. Clin Chest Med 1991;12:643-58. 